\title{
Contando la realidad con números. Ciclo de Mejora en la asignatura Estadística Avanzada.
}

\section{Telling the reality whit numbers. An improvement cycle in Advanced Stitistics subject.}

LUIS ANDRÉS ZAMBRANA

Universidad de Sevilla, Departamento de Economía Aplicada II.

lazambrana@us.es

http://orcid.org/0000-0003-3606-7816

DOI: http://dx.doi.org/10.12795/9788447231003.077

Pp.: 1654-1676 


\section{Descripción del contexto de la intervención}

Como ya hiciera en los dos cursos precedentes, el Ciclo de Mejora en el Aula correspondiente a 2020/2021 lo aplicaremos en la asignatura Estadística Avanzada, de segundo curso del Grado en Administración y Dirección de Empresas, este curso sería, por tanto, la tercera edición consecutiva de implementación de esta metodología. Como novedad, amén de las que se introducirán más adelante en lo que a contenidos se refiere, esta edición abarca siete de los ocho grupos en los que se imparte la asignatura en el grado de Administración y Dirección de Empresas, así como en los dos grupos del grado de Marketing e Investigación de Mercados. Como en cursos anteriores, se trata de ciclos de asignatura completa. Lo que, sin duda alguna, nos lleva a afirmar que la metodología de innovación docente iniciada tres cursos atrás, se consolida en la docencia de la asignatura Estadística Avanzada impartida en los grados antes referidos.

Estadística Avanzada es una asignatura cuatrimestral, de 6 créditos, impartida en 4 horas semanales, agrupada en bloques de 2 horas. Se trata de la segunda asignatura, contemplada en el plan docente del grado, de la disciplina Estadística, en el primer curso del Grado los estudiantes ya debieron superar la asignatura Estadística (con contenidos de Estadística Descriptiva), por tanto, deben estar familiarizados con muchos de los conceptos que forman parte de los contenidos que vamos a trabajar durante el curso.

La materia impartida, si la consideramos en grandes bloques, incluiría tres, a saber: Teoría de la Probabilidad y Variables Aleatorias, Teoría de Muestras e Inferencia Estadística (Estimación y Contrastes de Hipótesis) (Cano, 2018).

Ciclos de Mejora en el Aula (2020). Experiencias de Innovación Docente de la US Esta obra se distribuye con la licencia Creative Commons 
El primer CIMA en esta asignatura -completa- lo llevé a cabo en el curso 2018-2019, en dos de los ocho grupos del 2 o curso del Grado, como ya señalé en ediciones anteriores (Andrés, L. 2019), Estadística Avanzada, venía siendo una de las asignaturas con mayor tasa de fracaso en el Grado (más del $40 \%$ de los matriculados eran repetidores), a mi entender, y sin entrar en más detalles, la razón que lo explica es la abstracción de sus contenidos y la dificultad para que lo relacionen con la realidad, a lo que he de sumar el relato que trasmiten las sucesivas promociones de estudiantes sobre la dificultad de superar las asignaturas, lo que acaba etiquetando las mismas en función de lo que ellos consideran el "grado de dificultad". En este sentido "Estadística Avanzada" viene acompañada de la etiqueta de "las asignaturas más difíciles de la carrera", lo que a mi entender condiciona, en gran medida, la actitud con la que el estudiante afronta el aprendizaje de una materia, convirtiéndose en una barrera.

Sin embargo, los resultados obtenidos tras la implementación del primer ciclo de mejora, curso 2018/2019, podemos afirmar que fueron sustancialmente exitosos, consiguiendo romper la dinámica de suspensos y absentismo que caracterizaba la asignatura.

El segundo, curso 2019-2020, ocupó tres de los ocho grupos de la asignatura, confirmando la tendencia apuntada en el curso anterior y ofreciendo resultados aún mejores, como mostraremos más adelante.

En la tabla 1 mostramos los resultados obtenidos en los dos ciclos de mejora docente de las asignaturas completas:

Ciclos de Mejora en el Aula (2020). Experiencias de Innovación Docente de la US Esta obra se distribuye con la licencia Creative Commons 
Tabla 1. Comparativa de resultados CIMA 2018/2019 $-2019 / 2020$

\begin{tabular}{|l|c|c|c|c|}
\cline { 2 - 5 } \multicolumn{1}{c|}{} & \multicolumn{2}{c|}{ Curso 2018/2019 } & \multicolumn{2}{c|}{ Curso 2019/2020 } \\
\cline { 2 - 6 } \multicolumn{1}{c|}{} & Total & $\%$ & Total & $\%$ \\
\cline { 2 - 5 } \multicolumn{1}{c|}{ Núm. Grupos } & \multicolumn{2}{c|}{2} & \multicolumn{2}{c|}{3} \\
\cline { 2 - 6 } Matriculados & \multicolumn{2}{|c|}{143} & 171 & $81,4(1)$ \\
Presentados & 96 & $67,1(1)$ & 135 & $78,9(2)$ \\
Superaron & 75 & $78,1(2)$ & 36 & $21,1(2)$ \\
No superaron & 21 & $21,9(2)$ & \multicolumn{2}{c|}{6,4} \\
\cline { 2 - 6 } Nota media & \multicolumn{3}{|c|}{5,9} & \multicolumn{2}{c|}{} \\
\hline
\end{tabular}

(1)Sobre Matriculados

(2)Sobre Presentados

El aumento del número de matriculados se debe a la extensión del CIMA a un tercer grupo. Sí es significativo el crecimiento de alumnos presentados, se trata de los datos relativos a la primera convocatoria ordinaria del curso, en el caso de esta asignatura la que tiene lugar en el mes de enero (final del primer cuatrimestre), este incremento se explica por la mayor participación de los estudiantes en la signatura, condición necesaria para superarla en evaluación continua y al convencimiento de que es más fácil entenderla trabajando que "empollarla" en grandes dosis y pequeños espacios de tiempo previo al examen. Otro logro significativo del CIMA es la subida de nota media.

Una de las novedades más importante que incluiremos en el nuevo CIMA de 2020 radica en el número de docentes que participaremos en el mismo. Todos los grados y dobles grados impartidos en la Facultad de Ciencias Económicas y Empresariales, incluyen la asignatura Estadística Avanzada que imparte nuestro departamento, Economía Aplicada II, le corresponden ocho grupos en el Grado de Administración y Dirección de Empresas (GADE), dos en el Doble Grado, en Administración y Dirección de empresas y

Ciclos de Mejora en el Aula (2020). Experiencias de Innovación Docente de la US Esta obra se distribuye con la licencia Creative Commons 
Derecho (GADED) y dos en el Grado de Investigación y Técnicas de Mercado (Marketing), para el curso 2020/2021 desarrollaremos CIMA de asignatura completa en siete grupo de GADE, uno de GADED y los dos de Marketing, es decir, incluirá diez de los doce grupos en los que se imparte, para ello contaremos con la implicación de los profesores:

- Victor Pérez León, profesor sustituto interino, recién incorporado al departamento y que ha mostrado gran interés en seguir la metodología.

- Antonio Cano Orellana, en fase preliminar del programa FIDOP, actualmente participa en la edición 2020 del curso GDU.

- Luis Andrés Zambrana, en fase de permanencia en el programa FIDOP, con dos CIMA de asignatura completa, actuará como coordinador del proyecto.

El contexto en el que está teniendo lugar este Ciclo está siendo muy distinto al que esperábamos cuando lo planificamos.

Los estudiantes comenzaron el curso siguiendo las clases en una modalidad que se dio en llamar de "semipresencialidad", consistente en asistir a clase una semana de cada tres, el resto habría de seguirla a través de la Plataforma de Enseñanza Virtual, donde hemos hecho uso de la herramienta "BlackBoard Collaborate", esto no es una cuestión menor para la implementación de nuestra metodología que, en gran medida, se basa en la construcción del conocimiento a partir del análisis de problemas reales trabajándolos de forma colectiva, con una importante carga, como veíamos en CIMA de cursos anteriores (Andrés L., 2018, 2019)

El curso académico comenzó la semana del 13 al 16 de octubre, la primera semana, por indicaciones de la dirección del centro, la dedicamos a la presentación de la

Ciclos de Mejora en el Aula (2020). Experiencias de Innovación Docente de la US Esta obra se distribuye con la licencia Creative Commons 
asignatura, con lo que a la fecha de cierre de este artículo han transcurrido siete de las 15 semanas que tiene el curso completo, es decir menos de la mitad del mismo, lo que deja por desarrollar la mayor parte del ciclo.

Si todo ello no fuera suficiente, del 16 de octubre al 7 de noviembre, quien escribe este capítulo hubo de someterse a aislamiento domiciliario por positivo en COVID19, acto seguido, el 9 de noviembre, por expreso mandato de la autoridad autonómica, toda la Universidad hubo de renunciar a la "semipresencialidad" y pasar a la docencia virtual $100 \%$, con los inconvenientes que ello conlleva y que antes apuntamos. Es decir, casi la totalidad de las sesiones se han desarrollado tras una pantalla de ordenador, sin contacto en vivo con los estudiantes.

\section{Diseño previo del Ciclo de Mejora Docente}

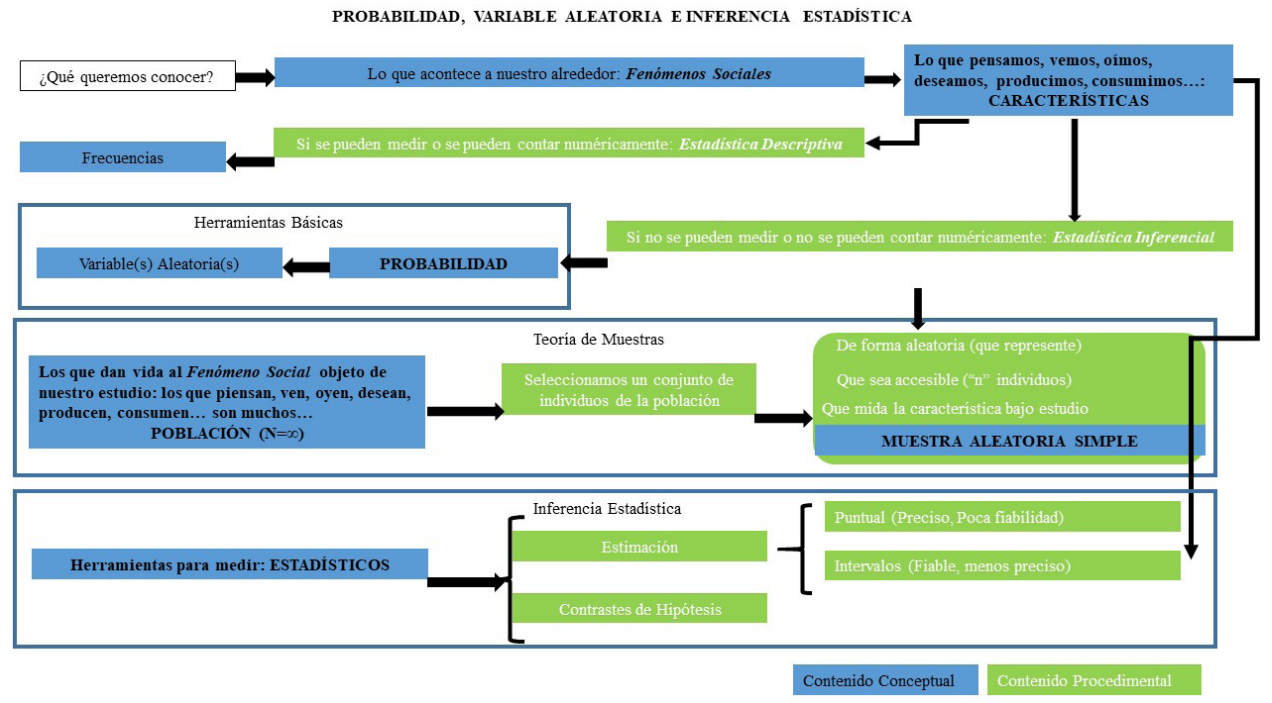

Figura 1. Mapa de contenido para el proceso de Inferencia Estadística

Ciclos de Mejora en el Aula (2020). Experiencias de Innovación Docente de la US Esta obra se distribuye con la licencia Creative Commons Reconocimiento-NoComercial-SinObraDerivada Internacional (CC BY-NC-ND 4.0.) 
El núcleo central de la asignatura es "la inferencia estadística", procedimiento mediante el cual seleccionamos una parte de un todo para observarlo y tratarlo con herramientas de estadística que nos llevarán a obtener conclusiones para el todo.

Nuestro objeto de estudio lo obtendremos a partir de la observación de un fenómeno social.

La experiencia de los cursos anteriores nos lleva al convencimiento de que la elección de, lo que en el mapa metodológico llamamos "problema general" y "problema específico" a partir de los cuales desarrollaremos las herramientas de Inferencia, no es una cuestión baladí. En los cursos anteriores elegimos como problema general "La turistificación de las grandes ciudades" (2018) y "La situación de Emergencia Climática" (2019); como problema específico, respectivamente, la estimación de la proporción de ciudadanos de Sevilla que percibían el turismo como una molestia y la proporción de estudiantes de la US que percibían vivir en una situación de Emergencia Climática.

Ambos problemas compartían la característica de "rabiosa actualidad", pero nos permitimos dudar de que fuesen cuestiones que "les preocupara", que hubiesen formado parte de sus planteamientos o de sus vivencias. Si bien con el transcurrir de las clases se familiarizaron, incluso en algunos casos sirvió para una toma de conciencia, lo cierto es que adolecía del interés inicial, a nuestro entender fundamental para la implicación en el aprendizaje.

Es por ello que en este curso nos planteamos enfrentar un problema general del que ellos forman parte y que, a nuestro entender, fuera más fácil de conectar con sus vivencias, en este sentido hemos elegido "El uso de las Redes Sociales", para aterrizar en un problema específico centrado en estimar la distribución del "tiempo que emplean los estudiantes de la Universidad de Sevilla en el uso de las Redes Sociales", sin que ello suponga renunciar

Ciclos de Mejora en el Aula (2020). Experiencias de Innovación Docente de la US Esta obra se distribuye con la licencia Creative Commons 
a la invitación a reflexionar sobre lo que hacemos y porqué (Manzano, 2012).

Aunque en el prediseño del CIMA proponíamos trabajar sobre la afectación del actual estado de pandemia a la actividad académica, tras un interesante debate en el aula, así como con el resto de compañeros de la asignatura, concluimos que podría ser una "sobredosis" de pandemia.

A partir del planteamiento inicial, definiremos las variables aleatorias que recojan las características que queremos estudiar, necesariamente han de ser características que poseen los individuos que forman una población (todos los estudiantes de la US). Como quiera que el tamaño de ésta es lo suficientemente grande (ya nos sirvió en 2019), como para que sea imposible al investigador acceder a todos ellos, seleccionaremos un subconjunto, al que llamaremos muestra, cuidando que sea representativo de toda la población (se lo encargaremos al azar, haciendo la selección de forma aleatoria). Observaremos en los individuos que forman la muestra la característica bajo estudio y le aplicaremos los procesos de Inferencia Estadística.

\section{Modelo metodológico}

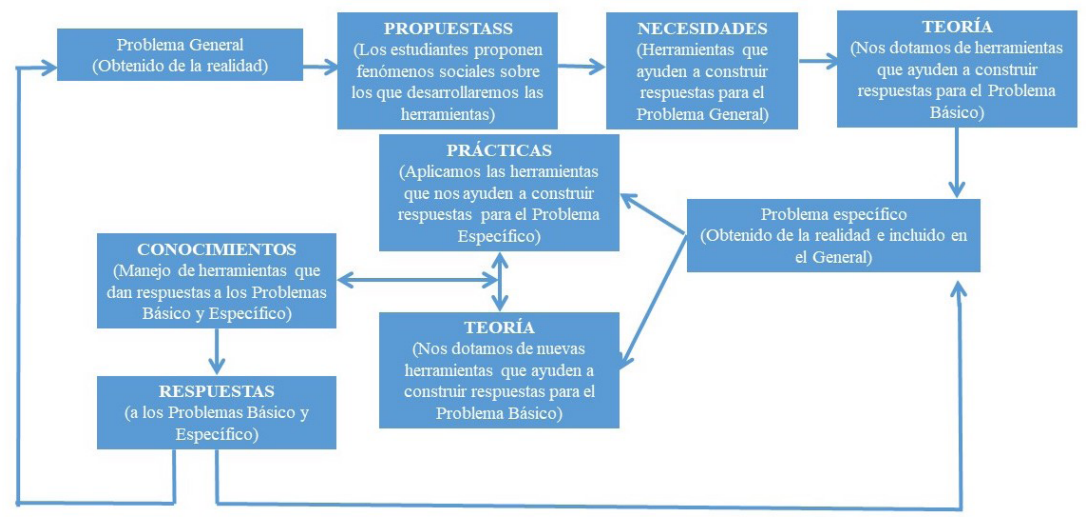

Figura 2. Modelo metodológico

Ciclos de Mejora en el Aula (2020). Experiencias de Innovación Docente de la US Esta obra se distribuye con la licencia Creative Commons 
El comienzo del ciclo de mejora docente coincidirá con el inicio del curso. Previamente los docentes implicados en el CIMA hemos consensuado el planteamiento inicial.

El objetivo final del curso es estimar los parámetros que determinan la distribución del "Tiempo que emplean diariamente los estudiantes de la Universidad de Sevilla al uso de las Redes Sociales", y esta propuesta nos llevará al planteamiento del problema específico ¿Aprendemos mejor con la docencia presencial? El desarrollo del ciclo de mejora se diseña como un proceso real encaminado a dar respuesta a dicha pregunta con números reales (Finkel 2008).

El proceso nos demandará herramientas de las que nos dotaremos en clases de teoría y requerirá un trabajo de campo que realizarán los estudiantes fuera del aula, como en el CIMA de 2019, tendrán que entrevistar a compañeros de la US de otros centros. Con el resultado obtenido aplicaremos, en sesiones prácticas, las herramientas de las que nos hemos dotado en teoría. El resultado final del CIMA es un trabajo real, desde el que se crea conocimiento que no está recogido en ninguna publicación previa y que está realizado con criterios profesionales.

\section{Aplicación del CIMA}

La trasmisión de lo que hacemos en clase es rápida y eficaz, es decir, los estudiantes de promociones anteriores comparten con los que le siguen sus experiencias en las clases, lo que les sirve para establecer criterios de elección de grupos y profesores, como no puede ser de otra manera el primer criterio de elección es "el porcentaje de aprobados en la asignatura", seguido por "el esfuerzo necesario para superarla".

Ciclos de Mejora en el Aula (2020). Experiencias de Innovación Docente de la US Esta obra se distribuye con la licencia Creative Commons 
Los CIMA de cursos anteriores, como ya pusimos de manifiesto anteriormente, han ofrecido porcentajes de aprobados muy por encima de lo que históricamente venía ocurriendo en esta disciplina, algo que para el estudiante es atractivo a la hora de elegir grupo, a lo que hemos de añadir que también les ha supuesto, no tanto una carga extra de trabajo, como una ordenación y una secuencia pautada de trabajo, lo que redunda en "facilidad" para superar la asignatura, pero también en la aprehensión de los contenidos.

Por estas razones, la demanda de pertenencia a los grupos que imparto es alta, lo que supone que algunos estudiantes que se quedan fuera acudan una vez empezado el curso, en la búsqueda de plaza para participar de esta metodología.

Todo lo expuesto en los párrafos anteriores tiene la "otra cara de la moneda", el que se pueda pensar que la asignatura es fácil de aprobar invocando a la ley del mínimo esfuerzo, algunos, como veremos en el análisis de resultados del CIMA 2019/2020, acuden pensando que con el simple hecho de entregar actividades es suficiente para aprobar. Por esta última razón, es de vital importancia la primera clase.

Desde el principio se hace tan necesario como útil un pacto en clase, en el que se aclare la tarea a desarrollar, como si el encargo de un trabajo profesional se tratara, y las recompensas que obtendremos por ello, de manera que aquellos que sigan la secuencia de actividades y trabajen, es muy probable que superen la asignatura sin gran dificultad (como muestran los resultados de cursos anteriores, resultados que expondremos en clase el primer día). El convencimiento de estos términos está, en gran medida, en la razón que explica la alta presencialidad en clase y la alta participación en actividades, aunque no siempre con el resultado deseado.

Ciclos de Mejora en el Aula (2020). Experiencias de Innovación Docente de la US Esta obra se distribuye con la licencia Creative Commons 
Una vez tenemos claro, todas las partes, a lo que nos vamos a dedicar y lo que vamos a obtener (superar o no la asignatura y un resultado real fruto de la aplicación de técnicas de investigación sobre un problema real), nos metemos en faena.

La primera de las tareas es la reflexión, el debate y la puesta en común sobre el problema real que guiará el curso y, por consiguiente, el CMD, como ya se señaló anteriormente este curso nos pareció adecuado trabajar sobre el uso de las redes sociales. Previo al inicio del trabajo propiamente dicho y a fin de promover la reflexión, el debate y puesta en común, proponemos el visionado del documental "El Dilema de las Redes Sociales" (Netflix, 2020).

Tras ver el documental, nuestra intención era que los estudiantes formaran grupos de 4, que durante 15 minutos debaten aquellas cuestiones que consideren relevantes de las que aparecen en el mismo y que las relacionaran con sus propias experiencias. Pasado ese tiempo un portavoz de cada grupo contaría al resto de la clase las conclusiones que hubieran obtenido y una vez expuestas todas las conclusiones intentamos consensuar aquellas cuestiones que mejor defina al colectivo. Con las respuestas de todos los grupos intentaremos construir un inventario de "afecciones" que nos brinde materiales para trabajar a partir de variables aleatorias, para posteriormente entrar en el mundo de la inferencia y estimar algún aspecto de esa realidad, aspecto previamente consensuado entre profesores y estudiantes en base a la relevancia, el interés y la capacidad que éste posea para ser susceptible de trabajarlo.

El trabajo en grupo en el escenario de semipresencialidad en el que comenzamos el curso se hacía muy complicado, más aún una vez retirados a la virtualidad total, por lo que los ejercicios de reflexión se pasaron al plano individual, lo que resta capacidad para confrontar, argumentar, recapacitar, tomar decisiones...

Ciclos de Mejora en el Aula (2020). Experiencias de Innovación Docente de la US Esta obra se distribuye con la licencia Creative Commons 
Al final del curso, con el trabajo de todos los estudiantes y los conocimientos que adquiramos en el manejo de las herramientas estadísticas tendremos nuestro estudio con la muestra adecuada, la explotación de la misma, las correspondientes estimaciones, contrastes y descripción de la metodología usada que avale nuestras conclusiones. En el curso 2020-2021, la media de estudiantes que están participando en el CIMA es de 65 por grupo, lo que supone alrededor de 450, en la medida en la que para el trabajo de campo cada uno se encargará de recopilar datos de diez estudiantes, dispondremos de una muestra de 4500 individuos, lo que sin duda es un trabajo de enorme potencial.

Tabla 2. Secuencia de actividades

\begin{tabular}{|c|l|}
\hline SEMANA & \multicolumn{1}{c|}{\begin{tabular}{c}
\multicolumn{1}{c}{ CONTENIDO } \\
Fase Metodológica: Presentación y Planteamiento del \\
Problema para trabajar
\end{tabular}} \\
$\begin{array}{c}\text { Semana 1 } \\
\text { Presentación de la asignatura, con base en dos pilares } \\
\text { sobre los que desarrollaremos los contenidos de la } \\
\text { misma. } \\
\text { 13-- El planteamiento de la misma en base a construir } \\
\text { la respuesta a un problema real. } \\
\text { 2.- El problema real al que daremos respuesta saldrá } \\
\text { de la reflexión que consensuemos en referencia al uso } \\
\text { que hacemos de las redes sociales } \\
\text { Se propone a los estudiantes el visionado del } \\
\text { documental "El Dilema de las Redes Sociales" (Netflix } \\
\text { 2020). }\end{array}$ \\
Recursos: Documental audiovisual
\end{tabular}

Ciclos de Mejora en el Aula (2020). Experiencias de Innovación Docente de la US Esta obra se distribuye con la licencia Creative Commons 
Fase Metodológica: Teórico-Práctica

Propuesta de trabajo individual: ¿Qué fenómenos relacionados con el uso que hacemos de las Redes Sociales podrían ser objeto de estudio para un investigador social? ¿ y de trabajo con las herramientas que nos provee la Estadística Inferencial? (Pregunta a usar en contraste, Actividad

Semana 2

(19-23/Octubre) 4 horas

01)

Las actividades han de depositarla en la Plataforma de Enseñanza, han de elaborar una propuesta por escrito y depositarla en tiempo y forma para trabajar en la siguiente clase.

Con las propuestas por ellos formuladas, analizamos ¿qué es objeto de estudio para un investigador social? y ¿qué podemos trabajar con las herramientas de la Estadística Avanzada

Recursos: Clase hablada y Power Point con resumen del trabajo de los estudiantes.

\begin{tabular}{|c|c|}
\hline \multirow{3}{*}{$\begin{array}{c}\text { Semana } 3 \\
\text { (26-30/Octubre) } \\
4 \text { horas }\end{array}$} & Fase Metodológica: Teórico \\
\hline & $\begin{array}{l}\text { Trabajamos las características que deben tener los } \\
\text { fenómenos objeto de nuestro trabajo. Comparamos los } \\
\text { resultados de las elecciones generales (11/2019) con } \\
\text { el último informe del CIS referido a la misma realidad } \\
\text { (10/2020), al objeto de ilustrar las diferencias entre } \\
\text { describir (Estadística descriptiva) e inferir (Estadística } \\
\text { inferencial): Trabajamos la Teoría de la Probabilidad y } \\
\text { Variables Aleatorias. } \\
\text { Los estudiantes han de establecer correspondencias } \\
\text { entre los conceptos vistos en clase y realidades } \\
\text { relativas al uso de redes sociales. Se les propone } \\
\text { que hagan una propuesta de estudio de una realidad } \\
\text { tratada con variable aleatoria continua y otra con } \\
\text { variable aleatoria discreta. }\end{array}$ \\
\hline & Recursos: Clase hablada y búsqueda en la Web. \\
\hline
\end{tabular}

Ciclos de Mejora en el Aula (2020). Experiencias de Innovación Docente de la US Esta obra se distribuye con la licencia Creative Commons Reconocimiento-NoComercial-SinObraDerivada Internacional (CC BY-NC-ND 4.0.) 
Semana 4 (3-6/Noviembre) 4 horas

Semana 5

(9-13/

Noviembre)

4 horas
Fase Metodológica: Problema Real/ Problemas Específicos

A partir de las propuestas de los estudiantes, elaboramos un listado/ranking y trabajamos en clase la viabilidad de abordar, con los recursos de los que disponemos, el estudio de las mismas. La clase tiene por objeto que los estudiantes reflexionen sobre la propuesta individual que cada uno haya hecho, La clase se convierte en un trabajo de "refinamiento" de sus propuestas.

A partir de la propuesta más repetida en todos los grupos, elevamos formalmente la que trabajaremos durante el curso, en base a una especie de "aclamación popular", consistirá en estimar los parámetros (media y varianza) de la variable aleatoria continua: "Tiempo que, diariamente, dedican los estudiantes de la US al uso de las Redes Sociales, medido en horas"

Recursos: Clase hablada y Power Point con los resultados de sus trabajos.

Fase Metodológica: Teoría y Prácticas

Exponemos las características de una distribución, especialmente la media y la varianza, protagonistas centrales del proceso de estimación.

En todo momento intentamos darle un sentido práctico y real a estas características, tirando de ejemplos con los propios alumnos: Sus notas en otras asignaturas, el dinero que tienen en el bolsillo...

Recursos: Clases Teóricas y encuesta en la clase, trabajo colectivo dirigido por el profesor. 
Vemos el desarrollo teórico de los modelos de distribución con los que trabajaremos, a saber, el modelo de Bernoulli y el modelo Normal. Para la Bernouilli realizamos un experimento en la propia clase. Para la distribución Normal conduzco el proceso para que ellos elaboren la campana de Gauss a partir de su percepción de la altura de las personas, como ya hiciera en cursos anteriores y que funcionó muy bien.

Semana 6

(16-20/

Noviembre) 4 horas

Finalmente formalizamos las distribuciones con el instrumental que necesitaremos, fundamentalmente un manual de Estadística con las tablas de cálculo que ha elaborado un compañero del Dpto. enfocado a los contenidos de la asignatura y nos descargamos de la web la aplicación gratuita, de gran utilidad para el cálculo de probabilidades y para el curso de Econometría que tendrán el 3o de GADE.

Se propone un ejercicio de manejo de las tablas y cálculo de probabilidades.

Recursos: Encuesta en la clase, trabajo colectivo dirigido por el profesor y manual-tablas estadísticas adaptado, descarga de la web de la aplicación "Gretl" Fase Metodológica: Teórico y Prácticas

Trabajo en clase con distribuciones normales, realizando prácticas con ejemplos reales que nos sirven para "contar" realidades de nuestro alrededor con números. Busco información acerca del uso de las redes sociales y, a partir de dicha información, construimos una distribución Normal.

Semana 7 Con la distribución que hemos construido elaboran, (23-28/ de forma individual, un informe respondiendo a noviembre) cuestiones concretas (Actividad 02). El objeto principal 4 horas de esta actividad es que sepan el significado de lo que contienen las tablas, que no se convierta en una búsqueda mecánica y que sepan "leer" en los números contenidos en dichas tablas. Finalmente generalizamos las distribuciones normales y trabajamos con vectores normales multivariantes.

Recursos: Búsqueda de información en la Web y manejo de tablas estadísticas. 
Semana 8

(30/ Noviembre a 4 de diciembre) 4 horas

(9 a 11)

Diciembre)

2 horas

Semana 10

(14 a 18/

Diciembre) 4 horas
Fase Metodológica: Prácticas

La primera parte la dedicamos a corregir la actividad 02. Con todos los matices hechos en la corrección de la actividad 02, sin previo aviso, en la segunda parte de la clase les propongo contar realidades con más de una dimensión con un doble objetivo, medir como han aprehendido los comentarios de la corrección en la actividad 02 y trabajar con realidades el vector aleatorio multinomial (Actividad 03)

Recursos: Búsqueda de información en la Web y trabajo en clase.

Fase Metodológica: Teoría

Esta semana, especialmente corta por el puente de diciembre, la dedicamos a la última "herramienta básica" del curso, previa a entrar en el objetivo propio del mismo, la Inferencia Estadística, se trata del

"Teorema Central del Límite". Les propongo un experimento para que comprueben el funcionamiento que subyace al teorema central del limite.

Recursos: Clase Magistral, Experimentación en clase.

Fase Metodológica: Practica

Prácticas en el aula con ejemplos reales.

Para el vector normal multivariante y el teorema central del límite trabajamos información relativa al uso de Redes Sociales, № de aplicaciones usadas, visitas... Los datos obtenidos se publican en la Enseñanza Virtual, parte lo trabajamos en clase y el resto lo trabajan en casa (Actividad 04).

Esta semana termina el primer bloque de la asignatura, al que hemos denominado Herramientas Básicas. Les dejo en Enseñanza Virtual un documento para que puedan practicar y fijamos la última semana de diciembre para la realización de una prueba en la que se les pedirá que manejen las mismas sobre la base de un caso real (Actividad 05)

Recursos: Información de la Web, trabajo en clase y trabajo en casa. 
Semanas 11 y 12 (21 y 22 /Diciembre y 7 y 8 de enero) 4 horas

Semana 13

(11 a 15 / Enero) 4 horas

Semana 14 (18 a 22 / Enero) 4 horas
Fase Metodológica: Contraste

Durante el primer bloque de dos horas los estudiantes realizaran una prueba, individual, en la que han de tratar un situación real con las herramientas trabajadas en el primer bloque de la asignatura (Probabilidad y Variable Aleatoria) El objeto de la prueba, además de usarla como medida de contraste, es el obligarlos a ordenar el inventario de las herramientas necesarias para la segunda y tercera parte, que son las que constituyen el objetivo de la asignatura (La construcción de conocimiento con el uso de la Inferencia Estadística). En el segundo bloque de clases analizamos el resultado de la prueba a fin de hacer las correcciones necesarias cara a la parte definitiva del curso.

Recursos: Prueba individual y correcciones

Fase Metodológica: Problema Real

Definimos con presición la característica a trabajar en el conjunto de todos los grupos en los que se ha puesto en marcha el ciclo.

Variable: "Tiempo diario que dedican los estudiantes de la US al uso de Redes Sociales" (Es una

distribución Normal, en la que intentaremos estimar llos parámetros necearios para poder trabajar con la misma, es decir, media y varianza).

Iniciamos el camino de Inferencia Estadística. En esta semana trabajamos la Muestra Aleatoria Simple, los Estadísticos y su funcionamiento en nuestro contexto de trabajo.

Recursos: Taller conceptual y prácticas

Fase Metodológica: Problema Real

Abordamos el proceso de Estimación y de Contraste de Hipótesis con la información que los estudiantes han obtenidoal realizar la muestra.

Los estudiantes elaboran el informe completo del proceso de inferencia realizado sobre el problema tratado.

Recursos: Taller conceptual y prácticas 
Semana 15

(25 a 29 / Enero)

4 horas
Fase Metodológica: Problema Real

Puesta en orden de todas las herramientas usadas

en el curso en referencia al problema trabajado,

dando sentido real a cada paso. Al final del proceso

tendremos números que nos sirven para contar

realidades que les acontece a ellos que, hasta el

momento, nadie ha formalizado con rigor científico.

Variable: "Tiempo diario que dedican los estudiantes

de la US al uso de Redes Sociales" (Es una

distribución Normal, en la que previamente se ha

estimado, con una muetra de más de 4500 individuos,

parámetros necearios para poder trabajar con la

misma, es decir, media y varianza)

Recursos: Taller de prácticas

\section{Evaluación}

Para la evaluación del ciclo de mejora usaremos las mismas herramientas que en ciclos anteriores.

A los estudiantes les proponemos entre 6 y 8 actividades que han de realizar, algunas en casa, otras en la propia clase, y que deben depositar en la plataforma de enseñanza virtual en tiempo y forma, como si formaran parte de un equipo de trabajo y estuvieran elaborando un "informe sobre la realidad en la que estamos trabajando". En ese camino a la construcción del informe final, cada paso ha de llevar las herramientas adquiridas en pasos anteriores, ese es el camino de aprendizaje. Cada actividad incluye o necesita del manejo de las herramientas en las que se centra la actividad anterior, con lo que conseguimos un trabajo escalonado, es por ello que las actividades sirven como "escaleras de aprendizaje".

En la medida en la que el trabajo desarrollado en casa es un continuo y no constituye elementos estancos, nos permite tomar el pulso a como el estudiante va aprendiendo y manejando las herramientas. De manera que vamos estableciendo la evaluación de cada uno, no como el

Ciclos de Mejora en el Aula (2020). Experiencias de Innovación Docente de la US Esta obra se distribuye con la licencia Creative Commons 
resultado de la media obtenida con la puntuación en cada actividad, si no con las habilidades mostrada en ese camino de aprendizaje recorrido, buscando en el error una oportunidad de aprendizaje en vez de una penalización que lastre el resultado final (Roger, 2002).

En el pacto inicial, el día de la presentación de la asignatura, acordamos que la nota final recogerá el recorrido que han tenido en las actividades en una progresión donde la última actividad (elaboración del informe) es la más importante ya que recoge todas las herramientas, desde la definición de la variable, el establecimiento de su recorrido hasta el proceso de estimación y contraste.

La actividad final consistirá pues en la construcción de todo el proceso de estimación sobre "El tiempo que diariamente dedican los estudiantes de la US al uso de las redes sociales", en la que habrán de especificar todo el camino recorrido desde el inicio hasta la obtención del "número" en cuestión, así como el significado de lo que hemos obtenido.

Los estudiantes que hayan recorrido el camino con éxito superarán la signatura, aquellos que no la superen, así como los que deseen mejorar su nota, dispondrán de un examen final que reproduce un problema específico, más o menos parecido al manejado durante el curso, con datos obtenidos de otras fuentes, para que transiten un camino con las mismas herramientas y secuencias trabajadas durante el curso.

En un análisis pormenorizado del trabajo de los estudiantes a lo largo del ciclo de mejora docente llevado a cabo en el curso anterior, 2019-2020, ya que, en el momento de cierre de este artículo, el ciclo que da lugar al mismo aún no llegó a la mitad, con lo que no es posible su

Ciclos de Mejora en el Aula (2020). Experiencias de Innovación Docente de la US Esta obra se distribuye con la licencia Creative Commons 
análisis. Como quiera que esta cuestión la venimos arrastrando curso tras curso, aprovechamos los resultados del curso anterior y hacemos la explotación de los mismos en este.

Hemos elaborado un análisis de correlaciones entre los resultados obtenidos por los estudiantes y la participación en el ciclo, la variable que mide la participación es el número de actividades realizadas de las 8 propuestas durante el curso, mientras que el aprendizaje alcanzado lo medimos mediante las calificaciones obtenidas. En principio cabe esperar una correlación positiva y fuerte, sin embargo, solo se cumple la primera de las características, una lógica correlación positiva, con un coeficiente de correlación de 0,46.

Si observamos los pares de puntos obtenidos en el cruce de ambas variables y los referenciamos a las líneas medias de éstas, obtenemos cuatro cuadrantes, señalados en rojo en la figura 3.

\section{Participación y calificaciones}

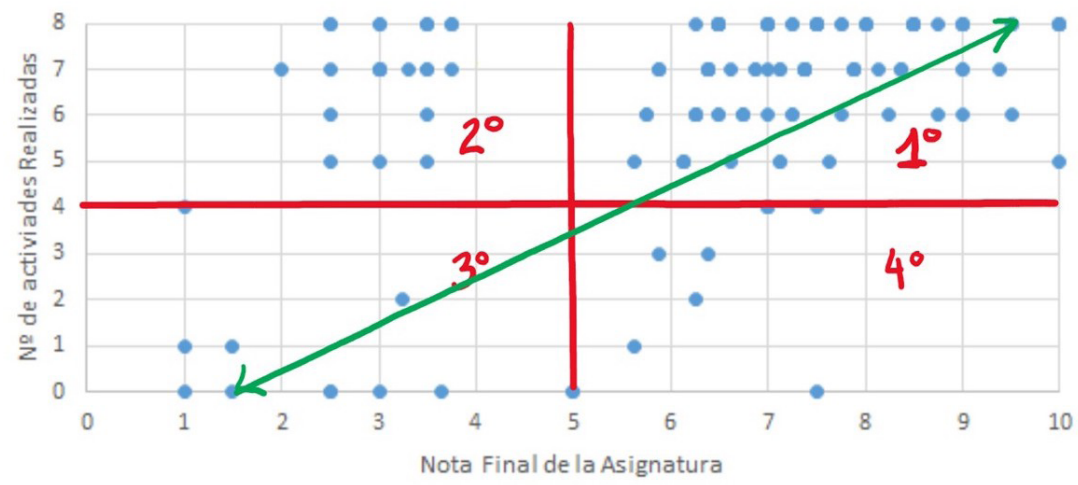

Figura 3. Análisis de correlaciones entre participación y resultados.

Los estudiantes que se encuentran en los cuadrantes impares, 10 y 30 - serían aquellos que han descrito el camino esperado, es decir los que más han participado obtuvieron Ciclos de Mejora en el Aula (2020). Experiencias de Innovación Docente de la US
Esta obra se distribuye con la licencia Creative Commons 
calificaciones más altas, mientras los que participaron en menor medida las calificaciones fueron más pobres. Este recorrido lo describiría la línea sobrepuesta en color verde en el gráfico, describiendo la tendencia esperada.

Sin embargo, nos encontramos una presencia importante de observaciones en el 2o cuadrante, donde se encontrarían un nutrido grupo de estudiantes que han seguido el ciclo, simple y llanamente, entregando actividades, desde el convencimiento que solo con esa actitud podrían superar la asignatura, sin acompañar el camino con trabajo y, sobre todo, con la comprensión de las herramientas. Podemos afirmar que son estudiantes que acudieron al "efecto llamada" del cambio de metodología al que aludía en el contexto del CMD, pero que nunca se llegaron a comprometer de verdad.

Los pares de punto que se sitúan en el 4으 cuadrante reflejan los estudiantes que se presentan a una prueba final, sin seguir la evaluación continua, pero superan la asignatura, opción esta que se ofrece desde el primer día y que es aceptada por estudiantes que por distintas razones (trabajo, repetidores...) la adoptan.

\section{Evaluación del CIMA}

La evaluación del ciclo se hace difícil por las razones expuestas en el contexto y reiteradas en el apartado anterior, sin embargo, no nos impiden alcanzar algunas conclusiones.

1. Sin que esta primera conclusión suponga escudarse en una argumentación repetida hasta la saciedad entre los docentes, un inconveniente difícil de subsanar para la implementación del ciclo es el elevado número de estudiantes que hay en cada grupo. Una participación por encima de los 60 estudiantes por 
grupo hace muy difícil el seguimiento del camino de aprendizaje de cada uno de ellos, así como la devolución de los resultados de sus trabajos/actividades en un plazo razonable de tiempo para que se pueda trabajar sobre ello. Inconveniente que se ve agravado por el elevado número de créditos que supone el actual encargo docente. Esta cuestión, a nuestro entender, es a todas luces incompatible con un plan de mejora docente en la Universidad.

2. Abstrayéndonos de las dificultades que impone el contexto y que no parecen tener una solución cercana, hemos de reconocer que el camino seguido en esta asignatura desde que empezamos a implementar los CMDs en el curso 2018/2019, es ilusionante. Cada año está abarcando a más docentes, más estudiantes, más grupos y ofreciendo mejores resultados, lo que sin duda es una carga anímica positiva para seguir el camino de innovación docente.

3. Los estudiantes que pasan por el CMD vienen de un contexto diametralmente opuesto al espíritu y la metodología de los mismos, un contexto que aunque pueda parecer que le pone más "obstáculos" para alcanzar el objetivo de superar las asignaturas, ellos se han entrenado y lo aceptan como su zona de confort, por lo que se hace necesario sacarles de esa situación. Es por ello que un grupo de docentes del grado estamos elaborando una experiencia piloto que intente una docencia transversal de las distintas asignaturas en torno a un problema real. De esta iniciativa esperamos escribir en futuras publicaciones. 
Palabras claves: Estadística Avanzada; Aprendizaje en Disciplinas Técnicas; Grados en Administración y Dirección de Empresas, Investigación y Técnicas de Mercado y Economía. Docencia Universitaria; Experimentación Docente Universitaria; Educación en Valores.

Key words: Advanced Statistics; Learning in Technical Disciplines; Degrees in Business Administration and Management, Research and Market Techniques and Economics. University teaching; University Teaching Experimentation; Education in values.

\section{Referencias bibliográficas}

Andrés, L. (2018). Ciclo de mejora en la asignatura Estadística Avanzada. Inferencia Estadística: estimación puntual de los parámetros de una distribución de probabilidad. Jornadas de Formación e Innovación Docente del Profesorado, 1, 1588-1605. Sevilla: Universidad de Sevilla

Andrés, L (2019). Ciclo de mejora en la asignatura Estadistica Avanzada. En E. Navarro-Medina y R. Porlán (Coord.), Ciclos de mejora en el aula. Año 2019. Experiencias de innovación docente de la Universidad de Sevilla (pp. 1731-1752). Sevilla: Editorial de la Universidad de Sevilla

Bain, K. (2007). Lo que hacen los mejores profesores universitarios. Valencia: Universitat de Valencia.

Cano, A. (2018). Curso de Estadística Avanzada Económica y Empresarial. Sevilla: Red de Impresión.

Finkel, D. (2008). Dar clase con la boca cerrada. Valencia: Universitat de Valencia.

Manzano, V. (2012). La universidad comprometida. Bilbao: Hegoa.

Morin, E., Roger E. y Domingo, R. (2002). Educar en la era planetaria. El pensamiento complejo como método de aprendizaje en el error y la incertidumbre humana. Valladolid: Universidad de Valladolid.

Porlán, R. (2017). Enseñanza universitaria. Cómo mejorarla. Madrid: Ediciones Morata

Ciclos de Mejora en el Aula (2020). Experiencias de Innovación Docente de la US Esta obra se distribuye con la licencia Creative Commons 